\title{
Antimicrobial peptides-loaded smart chitosan hydrogel: release behavior and antibacterial potential against antibiotic resistant clinical isolates
}

\author{
Nourollah Rezaei ${ }^{1,2}$, Hatef Ghasemi Hamidabadi ${ }^{1,2}$, Sadjad Khosravimelal ${ }^{3,4,5}$, Maria Zahiri ${ }^{6}$,
} Zahra Aliakbar Ahovan ${ }^{7}$, Maryam Nazm Bojnordi ${ }^{1,2}$, Behnaz Sadat Eftekhari ${ }^{8,9}$, Ali Hashemi ${ }^{7}$, Fatemeh Ganji ${ }^{3,4}$, Shahram Darabi ${ }^{10}$, Mazaher Gholipourmalekabadi ${ }^{3,4} *$

${ }^{1}$ Immunogenetic Research Center, Department of Anatomy \& Cell Biology, Faculty of Medicine, Mazandaran University of Medical Sciences, Sari, Iran

${ }^{2}$ Department of Anatomy \& Cell Biology, Faculty of Medicine, Mazandaran University of Medical Sciences, Sari, Iran

${ }^{3}$ Cellular and Molecular Research Centre, Iran University of Medical Sciences, Tehran, Iran

${ }^{4}$ Department of Tissue Engineering \& Regenerative Medicine, Faculty of Advanced Technologies in Medicine, Iran University of Medical Sciences, Tehran, Iran

${ }^{5}$ Department of Medical Biotechnology, Faculty of Allied Medicine, Iran University of Medical Sciences, Tehran, Iran

${ }^{6}$ The Persian Gulf Marine Biotechnology Research Center, The Persian Gulf Biomedical Sciences Research Institute, Bushehr University of Medical Sciences, Bushehr, Iran.

${ }^{7}$ Department of Microbiology, School of Medicine, Shahid Beheshti University of Medical Sciences, Tehran, Iran

${ }^{8}$ Department of Biomedical Engineering, Amirkabir University of Technology, Tehran, Iran.

${ }^{9}$ Department of Physiology and Institute for Medicine and Engineering, University of Pennsylvania, USA.

${ }^{10}$ Cellular and Molecular Research Center, Qazvin University of Medical Science, Qazvin, Iran

*Dr. Mazaher Gholipourmalekabadi (ORCID: 0000-0001-6287-6831)

Dr. Mazaher Gholipourmalekabadi

Department of Tissue Engineering \& Regenerative Medicine, Faculty of Advanced Technologies in Medicine, Iran University of Medical Sciences, Tehran 1449614535, Iran

Tel: (+98 21) 8862 2755; Fax: (+98 21) 88622533

E-mail: mazaher.gholipour@iums.ac.ir; mazaher.gholipour@gmail.com

\section{Abstract}

In this study, we synthesized thermo-responsive chitosan (TCTS) hydrogels, and loaded with different concentrations of antimicrobial peptide (AMP) $\left(0,4,8\right.$ and $\left.16 \mu \mathrm{g} \cdot \mathrm{ml}^{-1}\right)$ to fabricate an antibacterial wound dressing against resistant clinical isolates. Physico-chemical properties, release behavior, cytobiocompatibility and antibacterial activity of the AMP-TCTS hydrogels against standard strain and resistant Acinetobacter baumannii were fully determined in vitro. The TCTS-40\% $\beta$-glycerolphosphate hydrogels showed a gelation time of $15 \mathrm{~min}$ at $37{ }^{\circ} \mathrm{C}$. $80 \%$ weight loss at day 35 with no changes in $\mathrm{pH}$ value was observed. AMP-TCTS hydrogels showed a burst release of AMP (around 40\%) at day 1, and a controlled release up to day 7. A dramatic water uptake was observed at first $4 \mathrm{~h}$, and then continued for $10 \mathrm{~h}$ in a steady manner. All the AMP-TCTS hydrogels showed excellent cytobiocompatibility for human fibroblasts. The TCTS showed no antibacterial activity against both standard strain and clinical isolates. All the AMPTCTS hydrogels had strong antibacterial activity against standard strains, but only $16 \mu \mathrm{g} . \mathrm{ml}^{-1}$ showed antibacterial behavior against resistant A. baumannii. Our results strongly suggest the 16 $\mu \mathrm{g} \cdot \mathrm{ml}^{-1}$ AMP-TCTS hydrogel as an excellent antibacterial wound dressing against resistant $A$. baumannii, and now promises to proceed with pre-clinical investigations.

Keywords: chitosan; hydrogel; thermos-responsive; resistant bacteria, post-wound infection; wound dressing; 